\title{
Outcome of patients with stage IV high-risk Wilms tumour treated according to the SIOP2001 protocol: a report of the SIOP Renal Tumour Study Group.
}

Pasqualini $\mathrm{C}^{1}$, Furtwängler $\mathrm{R}^{2}$, van Tinteren $\mathrm{H}^{3}$, Teixeira $\mathrm{RAP}^{4}$, Acha $\mathrm{T}^{5}$, Howell $\mathrm{L}^{6}$, Vujanic G.M. ${ }^{7}$, Godzinski $\mathrm{J}^{8,9}$, Melchior $\mathrm{P}^{10}$, Smets $\mathrm{A}^{11}$, Coulomb-L'Hermine $\mathrm{A}^{12}$, Brisse $\mathrm{H}^{13}$, Pritchard-Jones $\mathrm{K}^{14}$, Bergeron $\mathrm{C}^{15}$, de Camargo $\mathrm{B}^{16}$, van den Heuvel-Eibrink $\mathrm{MM}^{17,18}$, Graf $\mathrm{N}^{2}$, Verschuur A.C. ${ }^{19}$

${ }^{1}$ Children and Adolescents Oncology Department, Gustave Roussy, Villejuif, France claudia.pasqualini@gustaveroussy.fr

${ }^{2}$ Department of Pediatric Haematology/Oncology, Saarland University Hospital, Homburg, Germany rhoikos.furtwaengler@uks.eu; graf@uks.eu

${ }^{3}$ Biometrics Department, Netherlands Cancer Institute, Amsterdam, The Netherlands h.v.tinteren@nki.nl

${ }^{4}$ Instituto do Tratamento do Câncer Infantil, Department of Pediatrics, São Paulo University, São

Paulo, Brazil

rapteixeira@uol.com.br

${ }^{5}$ Hospital Materno-Infantil "Carlos Haya”, Department of Pediatrics, Malaga, Spain tomas.acha.sspa@juntadeandalucia.es

${ }^{6}$ Department of Oncology, Alder Hey Children's NHS Foundation Trust, Liverpool, United Kingdom lisa.howell@alderhey.nhs.uk

${ }^{7}$ Department of Pathology, Sidra Medicine, Doha, Qatar

gvujanic@sidra.org

${ }^{8}$ Department of Pediatric Surgery, Marciniak Hospital Wroclaw, Wroclaw, Poland

${ }^{9}$ Department of Pediatric Traumatology and Emergency Medicine, Medical University, Wroclaw,

Poland

jgodzin@wp.pl

${ }^{10}$ Department of Radiation Oncology, Saarland University Hospital, Homburg, Germany patrick.melchior@uks.eu

${ }^{11}$ Department of Radiology and Nuclear Medicine, Academic Medical Center, Amsterdam, The Netherlands

a.m.smets@amc.uva.nl

${ }^{12}$ Department of Pathology, Hospital d'Enfants Armand Trousseau, Paris, France aurore.coulomb@aphp.fr

${ }^{13}$ Department of Radiology, Curie Institut, Paris, France herve.brisse@curie.fr

${ }^{14}$ Great Ormond Street Institute of Child Health, University College London, London, UK k.pritchard-jones@ucl.ac.uk

${ }^{15}$ Pediatric Onco-Haematology Department, Centre Leon Berard, Lyon, France

christophe.bergeron@ihope.fr

${ }^{16}$ Instituto Nacional do Cancer, Pediatric Onco-Haematology, Rio de Janeiro, Brazil

bdecamar@terra.com.br

${ }^{17}$ Oncology, Princess Maxima Centre for Pediatric Oncology, Utrecht, The Netherlands

${ }^{18}$ Dutch Childhood Oncology Group, The Hague, The Netherlands

m.m.vandenheuvel-eibrink@prinsesmaximacentrum.nl

${ }^{19}$ Pediatric Onco-Haematology Department, Hopital de La Timone, AP-HM, Marseille, France arnauld.verschuur@ap-hm.fr 


\section{Corresponding author:}

Claudia Pasqualini, MD

Children and Adolescents Oncology Department

Gustave Roussy Cancer Campus

114 Rue Edouard Vaillant, 94800 Villejuif (France)

Tel: + 33 (0) 142114218

Fax: +33(0) 142116578

claudia.pasqualini@gustaveroussy.fr

\section{Acknowledgments/Sources of support}

This work was partly funded by the German CancerAid (Deutsche Krebshilfe, grant No: 502709-GR2), Association Léon Berard Enfant Cancéreux and Enfant et Santé (Albec), The DaDa Society (Nieuwerkerk a/d Ijssel, The Netherlands), The Paediatric Oncology Centre Society for Research (KOCR, Rotterdam, the Netherlands), Grupo Cooperativo Brasileiro para o Tratamento do tumour de Wilms (GCBTTW), Spanish Association Against Cancer (AECC), Cancer Research UK (grant refs: C1010/A2889 \& C1188/A8687) and National Cancer Research Network support to UK centers for data management, Parents of kids with cancer initiative of the Saarland (Elterninitiative Krebskranker Kinder im Saarland e.V., Germany) for continuous data management of GPOH-centres.

We thank all of the clinicians and families who participated in this study at all centers across 28 countries and all of the national childhood cancer groups for their support, in particular the Société Française des Cancers de l'Enfant (SFCE), Gesellschaft fur Paediatrische Onkologie und Haematologie (GPOH), Children's Cancer and Leukemia Group (CCLG), Spanish Society of Paediatric Haematology and Oncology (SEHOP), Sociedade Brasileira de Oncologia Pediatrica (SOBOPE), and Stichting Kinderoncologie Nederland (SKION). 


\section{Abstract}

Introduction: High-risk (HR) metastatic (stage IV) Wilms Tumours (WT) have a particular poor outcome. Methods: Here we report the results of HR (Diffuse Anaplastic (DA)- or Blastemal Type (BT)) stage IV WT treated according to the HR arm in the SIOP2001 prospective study. Results: From January 2002 to August 2014, 3559 patients with WT were included in SIOP2001 trial. Among the 525 patients (15\%) with metastatic WT, $74(14 \%)$ had stage IV HR-WT. Median age at diagnosis was 5.5 years (range: 1.4-18.3). Thirty-four patients (47\%) had BT-WT and 40 (53\%) had DA-WT. Five-year Event-Free-Survival (EFS) rates were $44 \pm 17 \%$ and $28 \pm 15 \%$ for BT-WT and DA-WT, respectively ( $\mathrm{p}=0.09$ ). Five-year Overall Survival (OS) rates were $53 \pm 17 \%$ and $29 \pm 16 \%$ for BT-WT and DA-WT, respectively $(\mathrm{p}=0.03)$. Metastatic complete response after preoperative treatment was significantly associated with outcome in univariate and multivariate analyses $(\mathrm{HR}=0.3 ; \mathrm{p}=0.01)$. Postoperative radiotherapy of metastatic sites might also be beneficial. Forty-three out of 74 patients experienced a relapse or progression predominantly in the lungs $(80 \%)$. The median time to relapse/progression after diagnosis was 7.3 months (range: $1.6-33.3$ ) and 4.9 months (range: $0.7-28.4$ ) for BT-WT and DA-WT, respectively $(\mathrm{p}=0.67)$. This is the first prospective evidence of inferior survival of stage IV BT-WT as compared to historical IR-WT. Survival of patients with stage IV DA-WT has not improved compared to the previous SIOP93-01study. Conclusion: These results call for new treatment approaches for stage IV HR patients. 


\section{Introduction}

The International Society of Pediatric Oncology (SIOP) strategy for Wilms Tumour (WT) is tailored to the patient based on overall tumour stage at diagnosis (localised, metastatic (stage IV), or bilateral (stage V) disease), histological risk group and local stage of the primary tumour after preoperative chemotherapy and nephrectomy (stage I-III), and response to preoperative treatment of metastatic or bilateral disease (1). With this approach, survival has risen to a current cure rate of more than $90 \%$ for patients with localized disease and intermediate- or low-risk (IR/LR) histology (2). Stage IV disease occurs in $12-20 \%$ of patients at diagnosis (3-5). The survival rates of stage IV patients reach $90 \%$ in case of IR/LR histology and metastatic complete response (m-CR) after preoperative chemotherapy and surgery. However, almost $20 \%$ of children with metastatic WT at diagnosis die (3). The negative impact of diffuse anaplasia (DA) on survival has been widely demonstrated (6-9). In patients with metastatic disease enrolled in the previous SIOP 93-01 trial, DA-WT had been associated with a lower 5-year event-free survival (EFS) compared with IR/LR histology (33.3\% vs $76.8 \%, \mathrm{p}<.001$; hazard ratio, $3.6 ; 95 \% \mathrm{CI}, 1.7$ to 7.6$)$, thus being confirmed as one of the most important prognostic factors in WT (3). The blastemal subtype (BT-WT) has been associated with poor outcome based on SIOP93-01 results $(10,11)$ and therefore defined and treated as "high-risk" (HR) histology in the prospective international SIOP2001 study (12). A recent report showed improved survival for localised BT-WT as a consequence of intensified treatment (13). We thus analysed whether this effect can also be documented in the stage IV cohort and present the outcome of patients with stage IV HR (DA- and BT-WT) WT treated according to the SIOP2001 protocol with an intensified postoperative schedule.

\section{Patients and methods}

\section{Patients}


Patients with metastatic BT- and DA-WT were prospectively included in the SIOP2001 study from 2002 to 2014. Clinical data were retrieved from the SIOP2001 database and through national coordinators and/or local centres. Written informed consent was obtained from all the patients or from at least one parent/legal guardian. The SIOP2001 study was submitted to the European Clinical trial register (EudraCT no: 2007-004591-39). Ethical approval was obtained in all countries.

\section{Treatment}

Treatment for stage IV HR WT in SIOP 2001 is summarized in Figure 1.

Preoperative treatment consisted of a 6-week regimen (AVD) as follows: vincristine weekly $\left(1.5 \mathrm{mg} / \mathrm{m}^{2}, \max 2 \mathrm{mg}\right.$ ), actinomycin D (45 micrograms $/ \mathrm{kg}$; $\max 2 \mathrm{mg}$ ) at week 1,3 and 5 , and doxorubicin $\left(50 \mathrm{mg} / \mathrm{m}^{2}\right)$ at week 1 and $5(2 / 3$ reduction applicable to patients $<12 \mathrm{~kg})$. A reassessment imaging of local tumour and metastatic sites was performed before tumournephrectomy after week 6 of preoperative treatment. Local staging and histology risk group was assessed according to the revised SIOP Classification for Renal Tumours (12).

In case of HR histology, postoperative treatment consisted of cyclophosphamide $\left(450 \mathrm{mg} / \mathrm{m}^{2}\right.$ for three consecutive days)/doxorubicin ( $50 \mathrm{mg} / \mathrm{m}^{2}$ one day) in postoperative week $1,7,19$ and 31 (maximal cumulative dose of anthracyclines $=300 \mathrm{mg} / \mathrm{m}^{2}$ ) alternating with etoposide $\left(150 \mathrm{mg} / \mathrm{m}^{2}\right) /$ carboplatin $\left(200 \mathrm{mg} / \mathrm{m}^{2}\right)$ for three consecutive days in postoperative week 4,10 , 13, 16, 22, 25, 28 and 34. Flank radiotherapy (RT) of 25.2 Gy (with 10 Gy boost in case of macroscopic residues or involved lymph nodes, according to physician's decision) was given to all stage III HR patients and to stage II patients only in case of DA histology. The entire peritoneal cavity was irradiated to a maximum of $21 \mathrm{~Gy}$ in case of intra-peritoneal dissemination. 
Metastasectomy was performed after nephrectomy whenever possible in case of persisting lesions. RT of metastatic sites (m-RT) such as lungs, brain and bone was recommended regardless of the response to chemotherapy. In case of pulmonary metastases, whole lung RT was applied to both lungs, for a total dose of 15 Gy with fractions of 1.5 Gy and a boost of 510 Gy on any residual disease after surgery. In case of extra-pulmonary metastases, RT was applied respecting the adjacent organs.

\section{Statistical analysis}

EFS and OS at 5 years after diagnosis were estimated by actuarial methods of Kaplan and Meier, and comparisons were made with the log-rank test. Univariate survival analysis was performed for metastatic burden $(<1$ site versus $\geq 2$ sites $)$, metastatic response after preoperative chemotherapy, stage of the local tumour and histology. A multivariable model was constructed including all variables that were tested univariately with a $p<0.2$, and based on the Cox proportional hazards model. Continuous variables were described as mean value \pm $\mathrm{SD}$ if the distribution was normal $(\mathrm{P}>0.05$, Kolmogorov-Smirnov), or as median and range value if the distribution was not normal. Comparisons by histology were made using the parametric and non-parametric test whenever appropriate. We used IBM SPSS Statistics 25.0.

\section{Results}

\section{Patients' characteristics}

From January 2002 to August 2014, 3559 patients with WT were included in SIOP2001 trial. Among the 525 patients (15\%) with metastatic WT, LR-, IR- and HR-subtypes were observed in $61(12 \%), 390(74 \%)$ and $74(14 \%)$ patients, respectively.

Among the 74 patient with stage IV HR-WT, BT-WT subtype occurred in 34 patients (46\%) and DA-WT in 40 patients (54\%). The characteristics of stage IV HR-WT cohort are listed in 
Table 1. Gender distribution was 1.2:1 (F:M). Median age at diagnosis was 5.5 years (range: 1.4-18.3). Local stage of the tumour was reported in 73/74 (one treatment related death before surgery) as following: stage I, II and III in $6(8 \%), 19(26 \%)$ and $48(66 \%)$ patients, respectively. The main site of metastases was lungs (96\%), which was the only involved metastatic site in 56/74 patients (76\%). "Liver only" metastases were reported in 3 patients (4\%). No significant differences were observed between the two HR-WT subtypes in terms of age, gender, local stage and site of metastasis. However, DA-WT had a strong trend to a higher rate of locally extended tumours (stage III) and metastases to sites other than lungs (Table 1).

\section{First line treatment}

First line treatment for each patient is reported in Supplementary Table 1.

All patients received AVD preoperative chemotherapy except two adolescents who presented with DA-WT, who had surgery before referral to a pediatric oncology department. Metastatic CR after preoperative chemotherapy was reported in 10/34 (29\%) and 2/38 (5\%) patients with BT-WT and DA-WT, respectively. Progressive disease (PD), mainly of metastasis, was reported during preoperative treatment in 6/38 (16\%) patients with DA-WT. All patients underwent nephrectomy except one 5-year old patient with BT-WT (post-mortem evaluation) and metastatic CR who died of actinomycin D-related sinusoidal obstruction syndrome $(\mathrm{SOS})$

Among the 65 patients who did not experience PD nor toxic-death during preoperative treatment and the 2 patient who underwent upfront surgery, data on postoperative chemotherapy and local RT were available in 63/67 (94\%) and 66/67 patients (98\%), respectively. Fifty-eight of them (89\%) started the postoperative treatment with HR regimen according to SIOP2001. Two patients with DA-WT received an alternative regimen as per 
physician's choice (Ifosfamide or Cyclophosphamide + Carboplatin + Etoposide - ICE/CCE regimens) (14). For unknown reasons, 3 patients received AVD regimen (BT-WT, n=1; DAWT, $n=2$ ), despite not being in metastatic CR. High-dose chemotherapy (HDC) followed by autologous stem cell rescue (ASCR) was administered in 6 patients (BT-WT, n=2; DA-WT, $\mathrm{n}=4$ ) as part of first line treatment. The administered HDC consisted of: HD Melphalan 200 $\mathrm{mg} / \mathrm{m}^{2}(\mathrm{n}=4)$, HD Carboplatin AUC $20+$ Etoposide $1 \mathrm{~g} / \mathrm{m}^{2}+$ Melphalan $180 \mathrm{mg} / \mathrm{m}^{2}(\mathrm{n}=1)$, and tandem HDC with Thiotepa $720 \mathrm{mg} / \mathrm{m}^{2}$ followed by HD Etoposide $1.8 \mathrm{~g} / \mathrm{m}^{2}+$ Melphalan $140 \mathrm{mg} / \mathrm{m}^{2}(\mathrm{n}=1)$.

Flank or abdominal RT was performed in 47/66 patients $(71 \%), 38$ stage III (BT-WT, n=18; DA-WT, $n=20)$ and 9 stage II (BT-WT, $n=4$; DA-WT, $n=5)$. Nineteen patients did not receive local RT for the following reasons: stage I (BT-WT, $n=3$; DA-WT, $n=3$ ), stage II and BTWT ( $\mathrm{n}=8)$, and early PD (BT-WT, $n=2 ;$ DA-WT, $n=3)$.

Data on m-RT were available in 63/67 patients (94\%). Thirty-three patients (52\%) received m-RT during first line treatment, 14 and 19 patients with BT-WT and DA-WT, respectively, regardless of the metastatic response. Median time to m-RT after surgery was 2.6 months (range: $0.5-8.6 \mathrm{mo})$. Nineteen patients $(\mathrm{BT}-\mathrm{WT}, \mathrm{n}=7$; DA-WT, $\mathrm{n}=12$ ) did not receive $\mathrm{m}-\mathrm{RT}$ as a first line treatment because of early postoperative metastatic progressive disease. Eleven patients (BT-WT, $n=8$; DA-WT, $n=3$ ) did not receive $\mathrm{m}-\mathrm{RT}$ for unknown reasons. Among them, 6 had achieved a metastatic CR by preoperative chemotherapy alone.

Globally, all patients received their postoperative treatment with only minor dose reduction of chemotherapy whenever needed, thus suggesting good tolerance of the whole treatment.

\section{Outcome}

At the time of the analysis, 33 patients were still alive, 19/34 and 14/40 patients with BT-WT and DA-WT, respectively. The median follow-up of censored cases was of 5.1 years $(95 \% \mathrm{CI}$, 
0.3 to 9.4 years). Five-year EFS rates were $44 \pm 17 \%$ and $28 \pm 15 \%$ for BT-WT and DA-WT, respectively ( $\mathrm{p}=0.09$; Figure 2 ). Five-year OS rates were $53 \pm 17 \%$ and $29 \pm 16 \%$ for BT-WT and DA-WT, respectively ( $\mathrm{p}=0.03$; Figure 3$)$.

The outcome according to preoperative response of metastases, local treatment and postoperative treatment in BT-WT and DA-WT is reported in Figure 4 and 5, respectively.

Forty-three out of 74 patients experienced a relapse or PD. The median time to relapse after diagnosis was 7.3 months (range: 1.6-33.3) and 4.9 months (range: 0.7 -28.4) for BT-WT and DA-WT, respectively $(\mathrm{p}=0.67)$. Thirty-two out of 43 patients $(74 \%)$ experienced a relapse/PD during treatment, 6 and 26 patients during preoperative chemotherapy and postoperative treatment, respectively. The site of relapse/progression was "lung only" in 35/43 patients (81\%), "liver only" in 3/43 (7\%), loco-regional in 3/43 (7\%), lung and liver in 1/43, and brain in $1 / 43$ patient $(2 \%)$. For the 67 patients who did not experience PD during preoperative treatment and who were alive after surgery, the administration of m-RT during first line treatment was associated with a significantly better 5-year EFS $(61 \pm 18 \%$ versus $13 \pm 13 \%$; $\mathrm{p}<0.001$, Figure 6). Excluding from the analysis those patients who experienced early postoperative progression $(<3$ months after nephrectomy, $n=7)$, the significant impact of $m$ RT was confirmed with a 5 -year EFS of $61 \pm 18 \%$ versus $17 \pm 17 \%(\mathrm{p}<0.001)$.

Three patients died of a treatment-related toxicity and $2^{\text {nd }}$ malignancy (SOS during preoperative treatment, $\mathrm{n}=1$; infection, $\mathrm{n}=1$; secondary leukaemia, $\mathrm{n}=1$ ).

In the univariate analysis, histology subtype, local stage and response of metastases after preoperative treatment had a significant impact on the outcome (Table 2, Figures 3 and 7). In the multivariate analysis, only response of metastases after preoperative treatment maintained a significant impact on survival $(\mathrm{HR}=0.3, \mathrm{p}=0.01$; Table 2$)$.

\section{Discussion}


The SIOP2001 study is the first international prospective protocol that considered both BT after chemotherapy and DA as HR factors. While the negative impact of DA had already been established (6-9), the introduction of blastemal predominance as a new HR histologic subtype was based on retrospective observations $(4,10)$. With the aim to increase their survival, patients with BT-WT included in the SIOP2001 protocol received an intensified treatment (HR regimen) compared to SIOP93-01 protocol where they were treated as IR-WTs.

Unfortunately, despite treatment intensification the outcome in stage IV BT-WTs is still significantly worse than those of stage IV IR-WTs enrolled in SIOP93-01 (5-year EFS of 77\%, 95\% CI: 71-83\%) (3). The negative impact of this HR histology confirms the results of the SIOP9/GPOH trial where, among the unilateral stage I-IV WT $(n=334)$, BT-WTs $(n=25)$ had a 5-year progression-free survival (PFS) of 58\%, slightly more than the 5-year PFS of $38 \%$ for the DA-WTs ( $n=21)$ but far from the $88 \%$ of WTs with IR histology (10).

Our current results for patients with stage IV DA-WTs are similar to those reported in patients enrolled in SIOP93-01 protocol, for whom a 5-year EFS of 33\% (95\% CI, 17-64\%) was reported with a similar chemotherapy regimen consisting of alternating etoposide/carboplatin and ifosfamide/epirubicin (or doxorubicin) courses for 34 weeks (maximal cumulative dose of anthracyclines of $400 \mathrm{mg} / \mathrm{m}^{2}$ ) (3). These results are comparable to those reported by the fifth National Wilms' Tumor Study (NWTS-5) (9). Patients with stage IV DA-WT (n=24/2596) were treated with a HR regimen based on vincristine, cyclophosphamide, etoposide, doxorubicine and radiotherapy (10.8 Gy to flank/abdomen + 12 Gy to lungs). In this report 4year EFS and OS rates of patients who received preoperative chemotherapy were $30.8 \%$ (95\% CI, 9.5- 55.4\%) and 44\% (95\%CI, 17-68\%), respectively.

In our HR cohort, the prognostic value of metastatic response to preoperative treatment has been confirmed. It is important to underline that only patients with DA-WT experienced PD before surgery, and they all died of disease. 
Postoperative m-RT seemed to have a significant impact on survival in our cohort. In order to take into account that some patients might have been excluded from m-RT because of early postoperative PD (selection bias), a specific analysis concerning only those patients without PD at least 3 months after nephrectomy was performed, showing that m-RT was still significantly associated with a better outcome. Considering that almost all the recurrences occurred in metastatic sites, especially in the lungs (80\%), current recommendations for HR stage IV in the Umbrella SIOP-RTSG2016 protocol are to perform radiotherapy of metastatic sites for all patients as early as possible, compatibly with local radiotherapy and metastasectomy whenever indicated.

These persisting poor survival rates underline the need for new strategies. The Children's Oncology Group (COG) AREN0321 study evaluated the activity of vincristine and irinotecan in a phase 2 window in newly-diagnosed patients with stage IV DA-WTs (15). Eleven out of 14 patients (79\%) had PR, and 3 had PD. The combination was well tolerated and has been incorporated into the current COG protocol for DA-WTs. The retrospective SIOP experience with irinotecan for relapsed WT was less encouraging, reporting, among the 4 DA- and 5 BTWT, one PR in a patient with BT-WT (16).

The role of upfront HDC for these patients is still unclear. Some encouraging results have been reported in very HR settings (17-19), but no randomized study has been performed yet. In our cohort, 6 patients received HDC followed by ASCR with heterogeneous consolidation regimens, thus precluding any definitive conclusion on the matter.

Based on these data, the SIOP-RTSG 2016 Umbrella protocol suggests an intensified regimen for stage IV HR WT based on a combination of vincristine, irinotecan, cyclophosphamide, carboplatin, etoposide, and doxorubicin (5). The choice of HDC is left at the discretion of the treating physician; data on safety and outcomes will be prospectively collected. 
Since $6 / 38(16 \%)$ of patients with DA-WT and preoperative treatment died after having experienced PD before surgery, early identification followed by an intensified treatment seems of major importance. Somatic mutations in TP53 are strongly associated with the development of anaplasia and with poorer survival (20). Originally thought to be pathognomonic for DA-WT $(21,22)$, the presence of TP53 mutations in BT and even in IR histology tumours with unfavourable evolution supports the addition of TP53 screening to the diagnostic workup, as a progression/aggressive marker (22). In the future, next generation sequencing-based methods could be employed at diagnosis to screen blood for TP53 mutations, allowing early intensification of preoperative chemotherapy $(21,23,24)$. Moreover, 1q gain, most frequent in BT-WT (25), and other potential molecular biomarkers will be prospectively assessed in the SIOP-RTSG 2016 Umbrella protocol, in order to better understand the biologic basis of resistant blastemal and DA.

In conclusion, the treatment of stage IV HR WT remains a challenge. The identification of novel agents is a priority. Collaborative research within the SIOP-RTSG and COG will offer the opportunity to develop new treatment paradigms by better understanding the biology of HR-WT.

\section{Conflict of interest statement}

The authors declare no conflict of interest.

\section{References}

1. SIOP Renal Tumour Study Group. Paediatric renal tumours: perspectives from the SIOPRTSG. Nat Rev Urol. 2017 Jan;14(1):3-4.

2. Pastore G, Znaor A, Spreafico F, Graf N, Pritchard-Jones K, Steliarova-Foucher E. Malignant renal tumours incidence and survival in European children (1978-1997): report from the Automated Childhood Cancer Information System project. Eur J Cancer. 2006 Sep;42(13):2103-14. 
3. Verschuur A, Tinteren HV, Graf N, Bergeron C, Sandstedt B, de Kraker J. Treatment of Pulmonary Metastases in Children With Stage IV Nephroblastoma With Risk-Based Use of Pulmonary Radiotherapy. Journal of Clinical Oncology. 2012 Oct;30(28):3533-9.

4. Graf N, van Tinteren H, Bergeron C, Pein F, van den Heuvel-Eibrink MM, Sandstedt B, et al. Characteristics and outcome of stage II and III non-anaplastic Wilms' tumour treated according to the SIOP trial and study 93-01. Eur J Cancer. 2012 Nov;48(17):3240-8.

5. van den Heuvel-Eibrink MM, Hol JA, Pritchard-Jones K, van Tinteren H, Furtwängler R, Verschuur AC, et al. Position paper: Rationale for the treatment of Wilms tumour in the UMBRELLA SIOP-RTSG 2016 protocol. Nature Reviews Urology. 2017 Oct 31;14(12):743-52.

6. Zuppan CW, Beckwith JB, Luckey DW. Anaplasia in unilateral Wilms' tumor: a report from the National Wilms’ Tumor Study Pathology Center. Hum Pathol. 1988 Oct;19(10):1199-209.

7. Vujanić GM, Harms D, Sandstedt B, Weirich A, de Kraker J, Delemarre JF. New definitions of focal and diffuse anaplasia in Wilms tumor: the International Society of Paediatric Oncology (SIOP) experience. Med Pediatr Oncol. 1999 May;32(5):317-23.

8. Green DM, Beckwith JB, Breslow NE, Faria P, Moksness J, Finklestein JZ, et al. Treatment of children with stages II to IV anaplastic Wilms' tumor: a report from the National Wilms’ Tumor Study Group. J Clin Oncol. 1994 Oct;12(10):2126-31.

9. Dome JS, Cotton CA, Perlman EJ, Breslow NE, Kalapurakal JA, Ritchey ML, et al. Treatment of Anaplastic Histology Wilms’ Tumor: Results From the Fifth National Wilms’ Tumor Study. Journal of Clinical Oncology. 2006 May 20;24(15):2352-8.

10. Weirich A, Ludwig R, Graf N, Abel U, Leuschner I, Vujanic GM, et al. Survival in nephroblastoma treated according to the trial and study SIOP-9/GPOH with respect to relapse and morbidity. Ann Oncol. 2004 May;15(5):808-20.

11. Reinhard H, Semler O, Bürger D, Bode U, Flentje M, Göbel U, et al. Results of the SIOP 93-01/GPOH trial and study for the treatment of patients with unilateral nonmetastatic Wilms Tumor. Klin Padiatr. 2004 Jun;216(3):132-40.

12. Vujanić GM, Sandstedt B, Harms D, Kelsey A, Leuschner I, de Kraker J, et al. Revised International Society of Paediatric Oncology (SIOP) working classification of renal tumors of childhood. Med Pediatr Oncol. 2002 Feb;38(2):79-82.

13. van den Heuvel-Eibrink MM, van Tinteren H, Bergeron C, Coulomb-L'Hermine A, de Camargo B, Leuschner I, et al. Outcome of localised blastemal-type Wilms tumour 
patients treated according to intensified treatment in the SIOP WT 2001 protocol, a report of the SIOP Renal Tumour Study Group (SIOP-RTSG). European Journal of Cancer. 2015 Mar;51(4):498-506.

14. Abu-Ghosh AM, Krailo MD, Goldman SC, Slack RS, Davenport V, Morris E, et al. Ifosfamide, carboplatin and etoposide in children with poor-risk relapsed Wilms' tumor: a Children's Cancer Group report. Ann Oncol. 2002 Mar;13(3):460-9.

15. Daw NC, Anderson JR, Hoffer FA, Geller JI, Kalapurakal JA, Perlman EJ, et al. A phase 2 study of vincristine and irinotecan in metastatic diffuse anaplastic Wilms tumor: Results from the Children's Oncology Group AREN0321 study. JCO. 2014 May 20;32(15_suppl):10032-10032.

16. Hol JA, van den Heuvel-Eibrink MM, Graf N, Pritchard-Jones K, Brok J, van Tinteren H, et al. Irinotecan for relapsed Wilms tumor in pediatric patients: SIOP experience and review of the literature-A report from the SIOP Renal Tumor Study Group. Pediatric Blood \& Cancer. 2018 Feb;65(2):e26849.

17. Ha TC, Spreafico F, Graf N, Dallorso S, Dome JS, Malogolowkin M, et al. An international strategy to determine the role of high dose therapy in recurrent Wilms' tumour. Eur J Cancer. 2013 Jan;49(1):194-210.

18. Kremens B, Gruhn B, Klingebiel T, Hasan C, Laws H-J, Koscielniak E, et al. High-dose chemotherapy with autologous stem cell rescue in children with nephroblastoma. Bone Marrow Transplant. 2002 Dec;30(12):893-8.

19. Furtwängler R, Nourkami N, Alkassar M, von Schweinitz D, Schenk J-P, Rübe C, et al. Update on relapses in unilateral nephroblastoma registered in 3 consecutive SIOP/GPOH studies - a report from the GPOH-nephroblastoma study group. Klin Padiatr. 2011 May;223(3):113-9.

20. Ooms AHAG, Gadd S, Gerhard DS, Smith MA, Guidry Auvil JM, Meerzaman D, et al. Significance of TP53 Mutation in Wilms Tumors with Diffuse Anaplasia: A Report from the Children's Oncology Group. Clinical Cancer Research. 2016 Nov 15;22(22):5582-91.

21. Treger TD, Chagtai T, Butcher R, Cresswell GD, Al-Saadi R, Brok J, et al. Somatic TP53 Mutations Are Detectable in Circulating Tumor DNA from Children with Anaplastic Wilms Tumors. Translational Oncology. 2018 Dec;11(6):1301-6.

22. Wegert J, Vokuhl C, Ziegler B, Ernestus K, Leuschner I, Furtwängler R, et al. TP53 alterations in Wilms tumour represent progression events with strong intratumour heterogeneity that are closely linked but not limited to anaplasia: TP53 in Wilms tumour. The Journal of Pathology: Clinical Research. 2017 Oct;3(4):234-48. 
23. Ludwig N, Nourkami-Tutdibi N, Backes C, Lenhof H-P, Graf N, Keller A, et al. Circulating serum miRNAs as potential biomarkers for nephroblastoma. Pediatr Blood Cancer. 2015 Aug;62(8):1360-7.

24. Jiménez I, Chicard M, Colmet-Daage L, Clément N, Danzon A, Lapouble E, et al. Circulating tumor DNA analysis enables molecular characterization of pediatric renal tumors at diagnosis. Int J Cancer. 2019 Jan 1;144(1):68-79.

25. Chagtai T, Zill C, Dainese L, Wegert J, Savola S, Popov S, et al. Gain of 1q As a Prognostic Biomarker in Wilms Tumors (WTs) Treated With Preoperative Chemotherapy in the International Society of Paediatric Oncology (SIOP) WT 2001 Trial: A SIOP Renal Tumours Biology Consortium Study. J Clin Oncol. 2016 10;34(26):3195-203.

\section{Legends}

Figure 1. Pre-operatory and post-operatory treatment of stage IV HR WT in SIOP2001

Figure 2. Event-free survival of stage IV HR WT according to histology subtype (BT-WT versus DA-WT)

Figure 3. Overall survival of stage IV HR WT according to histology subtype (BT-WT versus DA-WT)

Figure 4. Outcome according to preoperative response and postoperative treatment in BT-WT Figure 5. Outcome according to preoperative response and postoperative treatment in DA-WT Figure 6. Event free survival of stage IV HR WT according to radiotherapy of metastatic sites (m-RT) as component of first line treatment

Figure 7. Overall survival of stage IV HR WT according to metastatic response to preoperative chemotherapy before surgery (complete response, CR; partial response, PR; stable disease, $\mathrm{SD}$; progressive disease, $\mathrm{PD}$ )

Table 1. Main clinical features

Table 2. Univariate and multivariate analyses

Supplementary Table 1. First line treatment and outcome 
Supplementary Table 1. First line treatment and outcome

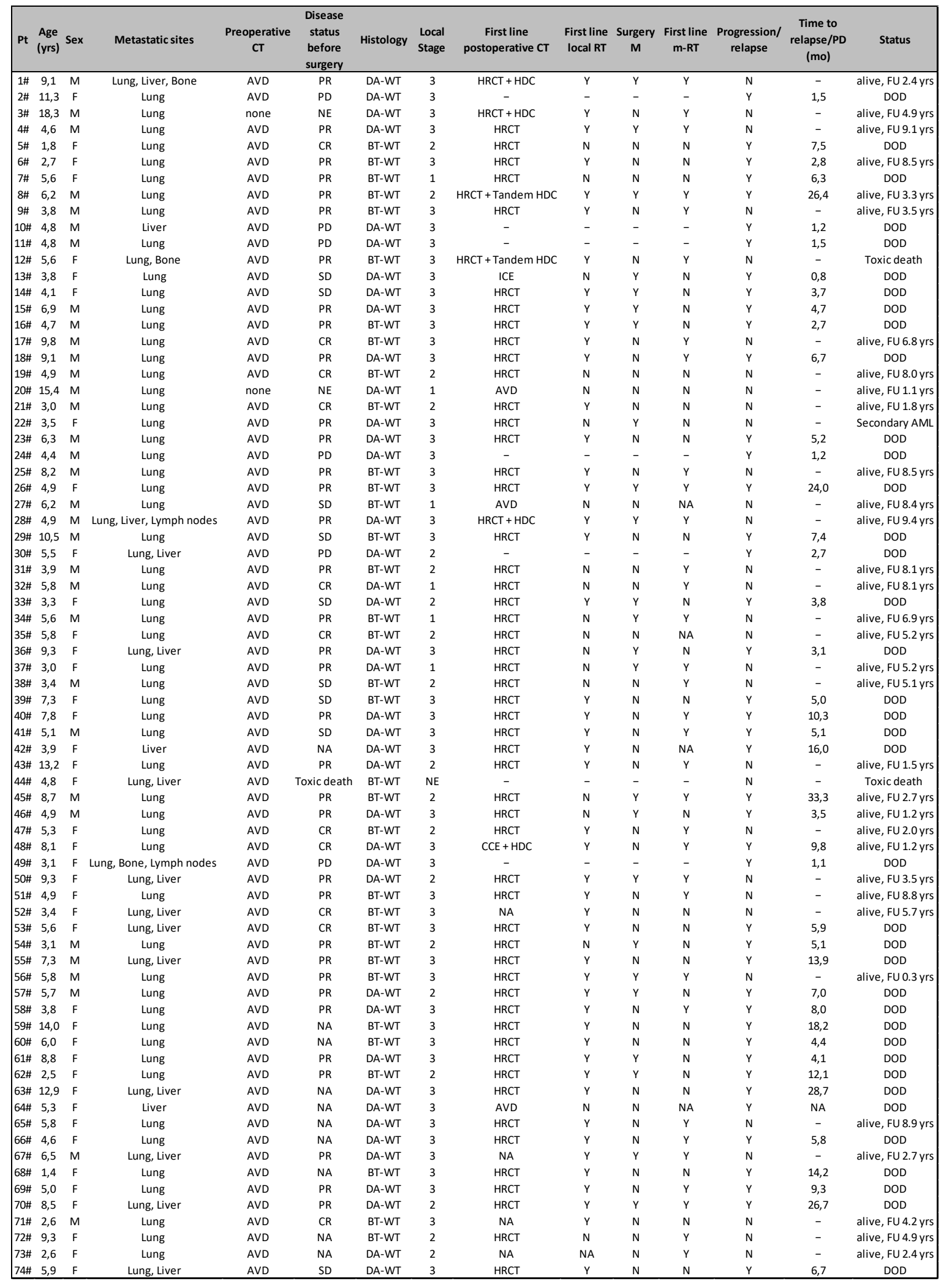

BT-WT: blastemal type wilms tumour; DA-WT: anaplastic wilms tumour; CT: chemotherapy; RT: radiotherapy; m-RT: radiotherapy of metastatic sites as first line postoperative treatment; CR: complete response; PR: partial response; SD: stable disease; PD: progressive disease; NA: not available; NE: not evaluated; HRCT: high-risk chemotherapy regimen; HDC: high dose chemotherapy with autologous stem cell rescue; FU: follow-up; DOD: died of disease; AML: acute myeloid leukaemia. 
Table 2: Univariate and multivariate analyses

\begin{tabular}{|c|c|c|c|c|}
\hline Variable & 5 -yrs OS & $\begin{array}{c}\text { Univariate } \\
(\mathrm{p})\end{array}$ & $\begin{array}{c}\text { Multivariate } \\
(\mathrm{HR})\end{array}$ & $\begin{array}{c}\text { Multivariate } \\
(\mathrm{p})\end{array}$ \\
\hline $\begin{array}{c}\text { Metastatic burden } \\
(1 \text { site vs } \geq 2)\end{array}$ & $\begin{array}{c}43 \pm 14 \% \\
30 \pm 25 \%\end{array}$ & 0.37 & - & - \\
\hline Histology & $53 \pm 17 \%$ & 0.03 & $1.5(0.7-3.0)$ & 0.30 \\
(BT-WT vs DA-WT) & $29 \pm 16 \%$ & 0.02 & $0.5(0.2-1.1)$ & 0.08 \\
\hline Local stage & $\begin{array}{c}80 \pm 35 \% \\
62 \pm 23 \%\end{array}$ & $29 \pm 13 \%$ & $0.3(0.1-0.8)$ & 0.01 \\
I vs II vs III & $\begin{array}{c}82 \pm 23 \% \\
\text { Preoperative metastatic } \\
\text { response }\end{array}$ & $\begin{array}{c}39 \% \\
\text { (CR vs PR/SD vs PD) }\end{array}$ & 0.001 & $0.3 \%$ \\
\hline
\end{tabular}

BT-WT: blastemal type wilms tumour; DA-WT: anaplastic wilms tumour; CR: complete response; PR: partial response; SD: stable disease; PD: progressive disease. 
Table 1. Main clinical features

\begin{tabular}{|c|cccc|}
\hline & $\begin{array}{c}\text { Whole } \\
\text { cohort } \\
\mathbf{n = 7 4}\end{array}$ & $\begin{array}{c}\text { Blastemal } \\
\text { Type } \\
\mathbf{n = 3 4}\end{array}$ & $\begin{array}{c}\text { Diffuse } \\
\text { Anaplasia } \\
\mathbf{n = 4 0}\end{array}$ & $\mathbf{p}$ \\
Median age (yrs) & 5.5 & 5.5 & 5.6 & 0.816 \\
Range & $(1.4-18.3)$ & $(1.4-14)$ & $(2.6-18.3)$ & \\
Sex (Female) & $41(55 \%)$ & $17(50 \%)$ & $24(60 \%)$ & 0.388 \\
Abdominal stage (n=73) & & & & \\
Stage I & $6(8 \%)$ & $3(9 \%)$ & $3(7 \%)$ & 0.805 \\
Stage II & $19(26 \%)$ & $12(36 \%)$ & $7(18 \%)$ & 0.068 \\
Stage III & $48(65 \%)$ & $18(55 \%)$ & $30(75 \%)$ & 0.067 \\
Site(s) of metastasis & & & & \\
Lungs only & $56(76 \%)$ & $29(85 \%)$ & $27(68 \%)$ & 0.075 \\
Lungs + Liver & $11(15 \%)$ & $4(12 \%)$ & $7(17 \%)$ & $\mathrm{NE}$ \\
Lungs + Bone & 1 & 1 & 0 & $\mathrm{NE}$ \\
Lungs + Liver + Bone & 1 & 0 & 1 & $\mathrm{NE}$ \\
Lungs + Bone + Lymph nodes & 1 & 0 & 1 & $\mathrm{NE}$ \\
Lungs + Liver + Lymph nodes & 1 & 0 & 1 & $\mathrm{NE}$ \\
Liver only & 3 & 0 & 3 & $\mathrm{NE}$ \\
\hline
\end{tabular}

NE: not evaluated due to low numbers; lymph nodes: extra-abdominal lymph nodes. 